\title{
SQUARE ROOTS IN BANACH *-ALGEBRAS
}

\author{
by F. F. BONSALL and D. S. G. STIRLING
}

(Received 23 April, 1970)

We give a simple proof of the lemma of Ford [1] on the existence of self-adjoint square roots in Banach *-algebras in which continuity of the involution is not assumed.

Let $A$ denote a real or complex Banach algebra and let

$$
\rho(a)=\inf _{n}\left\|a^{n}\right\|^{1 / n} \quad(a \in A) .
$$

Lemma 1. Let $a \in A$ and $\rho(a)<1$. Then there exists a unique element $x$ in $A$ for which $2 x-x^{2}=a$ and $\rho(x)<1$.

Proof. Since $\rho(a)=\inf |a|$, where the infimum is taken over all algebra-norms $|\cdot|$ equivalent to the given norm [2], we may choose such a norm $|\cdot|$ and real number $\eta$ with $|a|<\eta<1$. Let $C$ denote the least closed subalgebra containing $a$, and let $E=\{x \in C:|x| \leqq \eta\}$. Then $T$, given by

$$
T x=\frac{1}{2}\left(a+x^{2}\right),
$$

is a contraction mapping of $E$ into $E$, since the commutativity of $C$ gives

$$
|T x-T y|=\frac{1}{2}\left|x^{2}-y^{2}\right| \leqq \frac{1}{2}|x-y||x+y| \leqq \eta|x-y| \quad(x, y \in E) .
$$

Therefore there exists $x \in E$ with $2 x-x^{2}=a, \rho(x) \leqq|x|<1$.

Suppose now that $y \in A, 2 y-y^{2}=a$, and $\rho(y)<1$. Since $y$ commutes with $a$, and $x$ is a limit of polynomials in $a, y$ commutes with $x$. Therefore $\rho(x+y)<2$ and we may again choose an equivalent algebra-norm $|\cdot|{ }^{\prime}$ with $|x+y|^{\prime}<2$. But then the inequality

$$
|x-y|^{\prime}=\left|\frac{1}{2}\left(a+x^{2}\right)-\frac{1}{2}\left(a+y^{2}\right)\right|^{\prime} \leqq \frac{1}{2}|x+y|^{\prime}|x-y|^{\prime}
$$

gives $|x-y|^{\prime}=0$.

Lemma 2. (Ford [1]). Let $B$ be $a$ Banach *-algebra with $a \in B, a=a^{*}$ and $\rho(a)<1$. Then there exists $a$ unique $x \in B$ with $2 x-x^{2}=a, \rho(x)<1$ and $x=x^{*}$.

Proof. By Lemma 1 , there is a unique $x \in B$ with $\rho(x)<1$ and $2 x-x^{2}=a$. But $\operatorname{Sp} x^{*}=(\operatorname{Sp} x)^{*} ;$ so $\rho\left(x^{*}\right)=\rho(x)<1$, and $a=a^{*}=\left(2 x-x^{2}\right)^{*}=2 x^{*}-\left(x^{*}\right)^{2}$.

Therefore, by the uniqueness of $x, x=x^{*}$.

Remark. The proof does not use all the axioms of an involution. It applies to any mapping $x \rightarrow x^{*}$ such that $\rho\left(x^{*}\right) \leqq \rho(x)$ and $\left(2 x-x^{2}\right)^{*}=2 x^{*}-\left(x^{*}\right)^{2}$.

\section{REFERENCES} $521-522$

1. J. W. M. Ford, A square root lemma for Banach *-algebras, J. London Math. Soc. 42 (1967),

2. R. B. Holmes, A formula for the spectral radius of an operator, Amer. Math. Monthly 75 (1968), 163-166.

UNIVERSITY OF EDINBURGH 